\title{
Actividad polinizadora de insectos en huerto semillero clonal de Eucalyptus nitens (Deane et Maiden) Maiden, en Región del Biobío
}

\author{
Insect pollination in clonal seed orchards of Eucalyptus nitens \\ (Deane et Maiden) Maiden, in Region Biobio \\ Ximena Araneda D..$^{1 *}$, José Fernández . $^{1}$, Daniza Morales U. ${ }^{1}$, Jaime Espejo ${ }^{1}$
}

\begin{abstract}
RESUMEN
El objetivo de esta investigación fue determinar la presencia de polinizadores y el tiempo de permanencia en un huerto semillero clonal de Eucalyptus nitens. El estudio se realizó en el huerto semillero clonal Aguadas de Chumulco de propiedad de Forestal Mininco S.A., ubicado en la comuna de Mulchén, Región del Biobío. Para esto se realizó un ensayo en la temporada estival donde se determinó el tipo de polinizador en E. nitens mediante la captura de insectos con malla entomológica y posterior identificación por orden y familia mediante claves taxonómicas. Además, se tomaron registros de la actividad polinizadora de los insectos más predominantes durante una hora en tres períodos correspondientes a los tratamientos: mañana, medio día y tarde con siete repeticiones, registrando el tiempo de permanencia. Los resultados obtenidos indican que A. mellifera fue el insecto predominante en las flores de E. nitens durante el período estival con una participación del 91\%, el 9\% restante comprendió especies de los órdenes Hymenoptera y Diptera. Las visitas se concentraron durante las horas de la tarde, con mayor tiempo de permanencia A. mellifera durante la mañana y Bombus terrestris durante la tarde. En conclusión, los ordenes presentes sobre E. nitens fueron Hymenoptera y Diptera, con la especie A. mellifera seguido por B. terrestris, ambos con mayores visitas durante la tarde.
\end{abstract}

Palabras clave: polinizadores, Hymenoptera, Apis mellifera.

\begin{abstract}
The objective of this research was to determine the presence of pollinators and the time spent on a clonal seed orchard of Eucalyptus nitens. The study was conducted in clonal seed orchard Aguadas de Chumulco property Forest Mininco S.A., located in the commune of Mulchén, Bío Bío Region. For this trial was conducted in the summer where the type of pollinator E. nitens was determined by capturing insects with entomological net and subsequent identification by order and family by taxonomic keys. In addition, records pollination of the most prevalent insects were taken for 1 hour in three time periods corresponding to treatments: morning, noon and afternoon with seven replicates, recording the time spent. The results obtained indicate that $\mathrm{A}$. mellifera was the predominant insect in E. nitens flowers during the summer with a share of 91\%, the remaining 9\% understood species of Hymenoptera and Diptera orders. The visits were concentrated during the afternoon, with more time spent A. mellifera during the morning and in the afternoon Bombus terrestris. In conclusion, the orders present on E. nitens were Hymenoptera and Diptera, with the species A. mellifera followed by B. terrestris, both with more visits in the afternoon.
\end{abstract}

Key words: pollinators, Hymenopthera, Apis mellifera.

\section{Introducción}

Eucalyptus es un género de árboles de la familia de las Mirtáceas. Existen alrededor de 700 especies, la mayoría oriundas de Australia (Potts, 2004). Se encuentran distribuidas por gran parte del mundo debido a su rápido crecimiento, capacidad de adaptación a diferentes hábitats, frecuentemente se emplean en plantaciones forestales para la industria papelera, maderera o para la obtención de productos químicos, además de su valor ornamental (Santos et al., 2002; Pinto et al., 2004).
Del total de la superficie boscosa en Chile, 13,3 millones de hectáreas corresponden a bosques nativos y 2,41 millones de hectáreas a plantaciones forestales, donde tres especies concentran el 92,9\% de la superficie: Pinus radiata (60,9\%), Eucalyptus globulus $(22,4 \%)$ y Eucalyptus nitens $(9,6 \%)$ (INFOR, 2014). Una de las restricciones por las cuales $E$. nitens no ha sido masificada en Chile o en el extranjero es su escasa producción de semillas y su dificultad para ser propagado vegetativamente, además, presenta un alto grado de infertilidad y de hibridización, factores que pueden influir en la

\footnotetext{
1 Escuela de Agronomía. Universidad Católica de Temuco. Rudecindo Ortega 02950. Temuco, Chile.

* Autor para correspondencia: xaraneda@uct.cl
} 
disminución de las características genéticas deseables y vigor de estos (Rojas, 2014).

En Chile han avanzado significativamente los programas de mejoramiento genético de Eucalyptus, por lo tanto, el estudio en esta especie se presenta como una interesante alternativa (Barros, 2014). El adecuado conocimiento de la biología reproductiva, morfología floral, épocas de floración, polinizadores, etc., es fundamental para realizar técnicas de cruzamiento, ya que la relación de la entomofauna con los huertos de Eucalyptus causa impacto significativo sobre la diversidad, riqueza y distribución de especies (Majer y Recher, 1999).

Los huertos semilleros clonales son una plantación de clones seleccionados, donde el objetivo principal es la producción de semilla tanto de polinización abierta o controlada. Esto se puede realizar con un manejo intensivo para producir frecuentes cosechas con mayor ganancia genética en un lapso corto (Sanhueza, 2014). Además, del sitio elegido las labores de manejo en el tiempo son ineficientes si no se conocen las características de las flores y sus polinizadores en el área establecida, pues las especies vegetales dependen de las interacciones con los visitantes de las flores como polinizadores (Speight et al., 1999). Entre estos, los eucaliptos son polinizados principalmente por animales, con vectores que abarcan una amplia variedad de insectos, aves, marsupiales y algunas especies de murciélagos (Potts, 2004) y desde el punto de vista agronómico dentro de la polinización entomófila, la abeja (Apis mellifera $\mathrm{L}$.) es el insecto más eficiente y manejable, debido a que es el principal transportador de polen, que contribuye con más del $90 \%$ de la polinización cruzada (Klein et al., 2007).

Otro factor a considerar son las especies vegetales colindantes que influyen en la entomofauna existente en los Eucalyptus que a su vez debido a su metabolismo intervienen con un efecto alelopático que inhibe fuertemente el desarrollo de plantas herbáceas disminuyendo los insectos del sector y reduciendo la polinización (Souto et al., 2001).

Debido a lo anterior, el objetivo del presente trabajo fue determinar la presencia de polinizadores y el tiempo de visita en un huerto semillero clonal de E. nitens.

\section{Materiales y Métodos}

Esta investigación se llevó a cabo durante el período estival en el huerto semillero clonal Aguadas de Chumulco, propiedad de Forestal Mininco S.A., ubicado en la comuna de Mulchén, Región del Biobío, Chile ( $37^{\circ} 45^{\prime} 43^{\prime \prime}$ lat. Sur; $72^{\circ} 18^{\prime} 45^{\prime \prime}$ long. Oeste). La localidad se caracteriza por presentar temperaturas que varían en promedio durante enero en $26^{\circ} \mathrm{C}$ y una mínima en julio de $4{ }^{\circ} \mathrm{C}$. El régimen hídrico advierte una precipitación media anual de $1.008 \mathrm{~mm}$ (Tosso, 1985).

El material utilizado en el ensayo correspondió a distintos clones de árboles de E. nitens, establecido el 20 de octubre de 1995 en un diseño de bloques al azar con un total de 439 injertos, mayoritariamente del área de Victoria Central Australia, en una superficie de 1,1 hectáreas con una distancia de plantación de $6 \mathrm{~m} \mathrm{x} \mathrm{3,5} \mathrm{m.}$

Para este ensayo se seleccionaron siete clones con floración en más de dos ramas de forma aleatoria. Las plantas elegidas presentaron ramas con flores en distintos estadíos: con opérculo, con caída de opérculo y con flores. Luego, se determinó el tipo de polinizador presente en el huerto clonal mediante la captura de insectos con red entomológica. Estos fueron puestos en frascos con solución de Kalhe y rotulados con fecha y horario de captura, para su posterior identificación en laboratorio según claves taxonómicas a nivel de orden y familia.

Posteriormente, durante el período de floración se tomaron registros de la actividad polinizadora de los insectos más predominantes durante 1 hora en tres períodos: mañana (09:00 h a 10:00 h), mediodía (13:30 h a 14:30 h) y tarde (17:00 h a 18:00 h), junto con el tiempo de permanencia (en segundos) de estos insectos sobre las flores de $E$. nitens, utilizando un cronómetro.

Para la determinación del número de insectos dominantes y tiempo de permanencia de estos sobre la inflorescencia de $E$. nitens se utilizó un diseño completamente al azar con tres tratamientos (períodos de evaluación: mañana, mediodía y tarde) y siete repeticiones. Estos datos fueron sometidos a pruebas de análisis de varianza mediante test de KruskalWallis $(\mathrm{p} \leq 0,05)$ y T-Student $(\mathrm{p} \leq 0,05)$ utilizando el programa estadístico SPSS, versión 15.0.

\section{Resultados y Discusión}

La entomofauna encontrada durante el período de floración de E. nitens en la temporada de verano fue de 689 individuos pertenecientes a distintas especies de insectos (Tabla 1). Entre las principales órdenes de insectos se han reportado tres grandes grupos 
en plantaciones de Eucalyptus, como, Coleoptera, Lepidoptera e Hymenoptera (Cunningham et al., 2005). Asimismo, Sanzana et al. (2012) durante la realización de su ensayo registraron una ocurrencia de 741 individuos de un total de 17 especies de insectos visitantes florales, pertenecientes a cuatro órdenes, donde la mayor abundancia ocurrió en Coleoptera (301) e Hymenoptera (260), seguido de Diptera (179) y Lepidoptera (1).

En este estudio, dentro de los taxones colectados con mayor presencia, se encontraron Hymenoptera (667 individuos) y Diptera (22 individuos) similar a lo encontrado por Lopes et al. (2007) quienes recopilaron en su estudio 504 individuos, de los que la mayor cantidad pertenece a Hymenoptera (276), Lepidoptera (134) y Diptera (25). En este sentido, Klein et al. (2007) señalan que en la actividad polinizadora más del $80 \%$ es realizada por insectos donde las abejas contribuyen casi con el $80 \%$ de la polinización total. Como lo observado en este estudio, donde los resultados muestran a $A$. mellifera como la especie con mayor frecuencia de visitas en flores de E. nitens en el huerto semillero clonal, probablemente, por la presencia de néctar, el que es considerado la principal recompensa floral de los visitantes, al igual que el polen, razón por la cual se encuentran abejas, moscas y escarabajos para colectar el polen (Bhattacharya et al., 2005).

De los insectos observados en este estudio, el porcentaje de A. mellifera se estimó en un $91 \%$. El 9\% restante comprendió principalmente especies de los órdenes Hymenoptera y Diptera (Tabla 1), concordando con los resultados obtenidos por Hingston y Potts (1998), en una comunidad de flores en Tasmania donde A. mellifera fue la especie dominante. En tanto, en un estudio realizado en $E$. regnans por Griffin et al. (2009), clasificaron 33 grupos de polinizadores funcionales de acuerdo con la afinidad taxonómica y tamaño corporal, en donde el $92 \%$ de los insectos capturados eran dípteros, sin embargo, la mayoría de ellos eran pequeños y no contribuyen de manera significativa a la polinización, y además, las visitas efectuadas solo por insectos a la flor no da lugar a la producción ostensible de semillas (Hingston et al., 2004).

Por su parte, resultados obtenidos por Hingston et al. (2004) en cinco huertos semilleros localizados en Tasmania muestran que los vectores por excelencia en E. nitens fueron escarabajos (18), moscas (16), abejas (14), avispas (3), hormigas (2), y algunas pequeñas mariposas, así como también A. mellifera, y $B$. terrestris como visitantes comunes en flores de E. nitens, al igual que en el presente estudio, reportándose además el mayor número de especies de insectos herbívoros en plantaciones de Eucalyptus (Neves et al., 2012). Sin embargo, A. mellifera y $B$. terrestris producen menos del $7 \%$ de fertilización de semillas, debido a que la abeja es principalmente un consumidor de néctar, y un polinizador ineficiente y además deposita una alta proporción de polen endógamo (Rojas, 2014).

Otras especies de importancia del orden Hymenoptera correspondieron al género Bombus y Symphyta. En el género Bombus la especie encontrada fue $B$. terrestris con un $3,19 \%$. La especie del género Symphyta que correspondió a un 2,32\%, no fue clasificada. Mientras que, Sanzana et al. (2012) reportaron entre las especies más comunes en todo su estudio, solo Bombus sp. no presentando más del $1 \%$ de visitas florales.

Según Hingston et al. (2004) el tipo de vegetación (sotobosque) y flora adyacente a los huertos incrementan o disminuyen y seleccionan qué tipo de vectores se pueden encontrar, debido a que las comunidades de insectos en las plantaciones forestales son diferentes, tanto por un menor número de especies de insectos presentes y porque tienen pocas especies de insectos más abundantes (Cunningham et al.,

Tabla 1. Principales insectos polinizadores detectados en las flores de E. nitens.

\begin{tabular}{llcc}
\hline Orden & \multicolumn{1}{c}{ Familia } & Número de insectos & Porcentaje \\
\hline Hymenoptera & Apidae (A. mellifera) & 627 & 91 \\
& Apidae (B. terrestris) & 22 & 3,19 \\
& Tenthredinidae (Symphyta sp.) & 16 & 2,32 \\
& Vespidae & 2 & 0,29 \\
Diptera & Syrphidae & 12 & 1,74 \\
& Calliphoridae & 7 & 1,02 \\
& No clasificada & 3 & 0,44 \\
\hline
\end{tabular}


2005). Además, las actividades humanas reducen la complejidad del hábitat resultando en la pérdida de la diversidad de insectos y procesos ecológicos (Neves et al., 2012), y dependiendo de las comunidades de bosques y de si hay o no interacciones positivas o negativas entre estos tipos de hábitat, la comunidad de insectos que se desarrolla en una plantación dependerá de la cantidad de bordes en relación con el interior y por el aislamiento del hábitat, colonos y remanentes de bosque (Tscharntke et al., 2002).

En cuanto a la frecuencia de visitas de los insectos más predominantes en los diferentes horarios del día, se puede observar en la Figura 1 a A. mellifera como el principal polinizador en los tres períodos de evaluación. En las primeras horas de la mañana tanto A. mellifera como B. terrestris hacen visitas a la flor, aunque mucho menor que en horas de la tarde. A. mellifera realiza con mayor frecuencia visitas entre mediodía y tarde, lo que se debe al mayor requerimiento térmico para iniciar el vuelo de A. mellifera respecto de Bombus.

Con relación al tiempo de permanencia (Figura 2), no se obtuvieron diferencias significativas entre A. mellifera y B. terrestris sobre la flores de Eucalyptus y tampoco en los distintos horarios de medición (mañana, mediodía y tarde), no superando los 13 y 14 segundos de permanencia en la flor, tanto en abejas como en abejorro, respectivamente.
La permanencia en la flor dependerá del requerimiento energético del vector, por lo tanto, un menor tiempo de permanencia en la flor se asociaría a mayores necesidades energéticas, resultando en un mayor número de visitas de flores por unidad de tiempo para compensar sus requerimientos energéticos, siendo de mucha importancia en plantas de polinización entomófila, pues aumenta la polinización cruzada disminuyendo la autopolinización. Por lo tanto, Bombus al ser un insecto más grande, requeriría de un mayor gasto energético, debido a que debe mantener alta la temperatura de su cuerpo por lo que su permanencia es mucho menor en los períodos de mañana y mediodía, ya que al estar más tiempo sobre la flor comienza a bajar la temperatura de su cuerpo, y al succionar el néctar debe mantener en todo momento su tórax y abdomen calientes (Prys-Jones y Corbet, 1991) (Figura 2).

\section{Conclusiones}

Los principales órdenes presentes sobre las flores de E. nitens en el huerto semillero clonal Aguadas de Chumulco, ubicado en la comuna de Mulchén, Región del Biobío, fueron Hymenoptera y Diptera, destacando la especie $A$. mellifera seguido por B. terrestris.

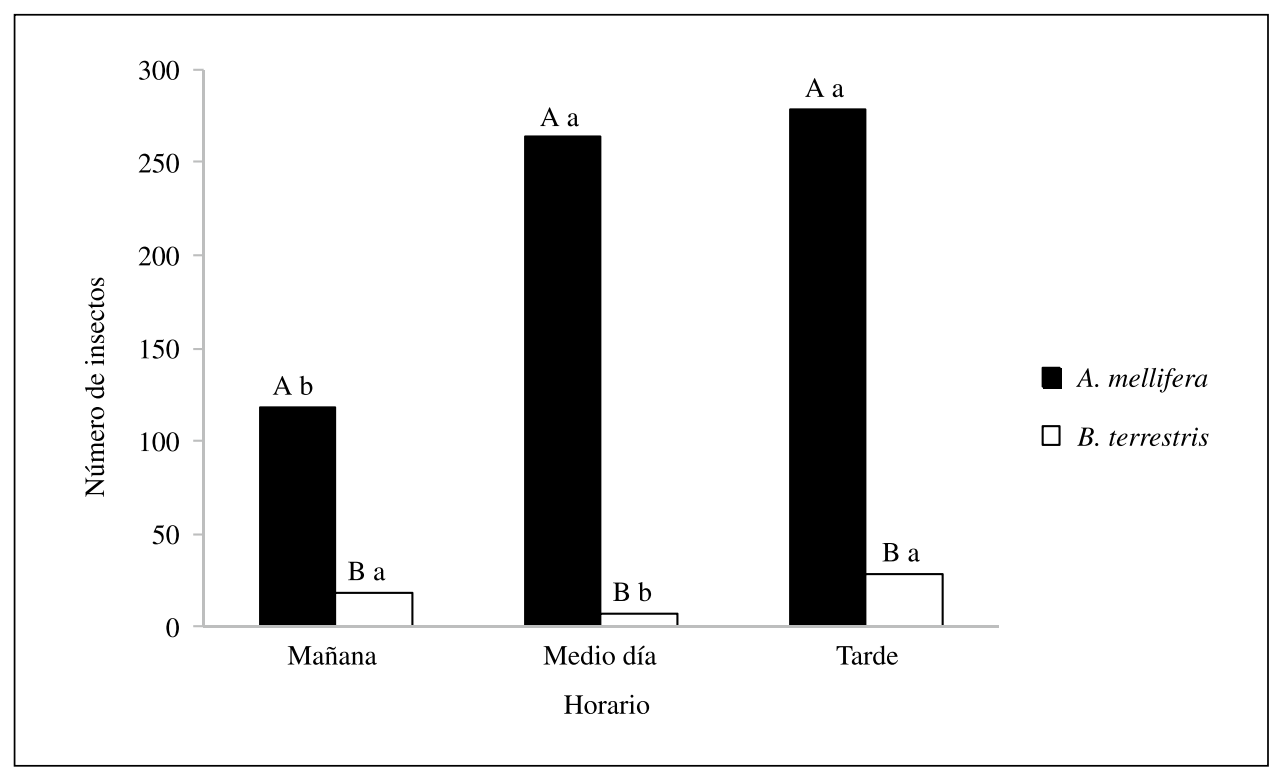

Figura 1. Número de insectos predominantes presentes en diferentes horarios sobre flores de E. nitens. Letras mayúsculas distintas en barras indican diferencias significativas entre especies en cada horario según T-Student $(\mathrm{p} \leq 0,05)$ y letras minúsculas indican diferencias significativas en los distintos horarios según test Kruskal-Wallis ( $\mathrm{p} \leq 0,05)$. 


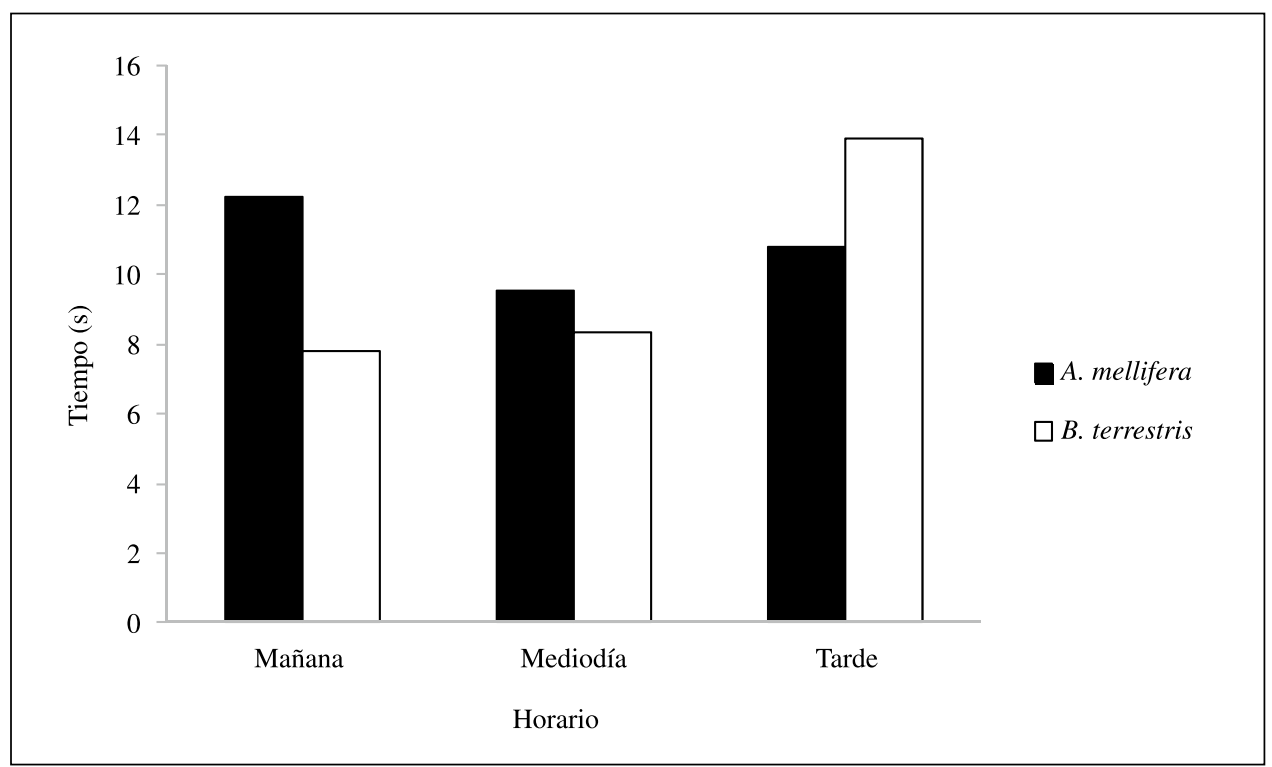

Figura 2. Tiempos promedios de permanencia de A. mellifera y B. terrestres sobre flores de E. nitens.

Los períodos de mayor visita de A. mellifera y $B$. terrestris son desde el mediodía hasta el final de la tarde en período estival.
Los tiempos de permanencia en las flores de E. nitens son mayores en A. mellifera durante las primeras horas del día hasta mediodía, y durante la tarde $B$. terrestris aumenta su permanencia.

\section{Literatura Citada}

Barros, $\mathrm{S}$.

2014. Los eucaliptos en Chile. In: Mejoramiento genético de eucaliptos en Chile. Ipinza, R.; Barros, S.; Gutiérrez, B.; Borralho, N. (eds.). INFOR-FIA. Santiago, Chile. pp. 65-88.

Bhattacharya, A.; Mondal, S.; Mandal, S.

2005. Pollinating agents of Eucalyptus citriodora Hook. Insects or Wind? Asian Journal of Plant Sciences, 4: 492-495.

Cunningham, S.; Floyd, R.; Weir, T.

2005. Do Eucalyptus plantations host an insect community similar to remnant Eucalyptus forest? Austral Ecology, 30: 103-117.

Griffin, A.; Hingston, A.; Ohmart, C.

2009. Pollinators of Eucalyptus regnans (Myrtaceae), the world's tallest flowering plant species. Australian Journal of Botany, 57(1): 18-25.

Hingston, A.; Potts, B.

1998. Floral visitors of Eucalyptus globulus subsp. globulus in eastern Tasmania. Tasforests, 10: 125-139.

Hingston, A.; Mcquillan, P.; Potts, B.

2004. Pollinators in seed orchards of Eucalyptus nitens INFOR.

(Myrtaceae). Australian Journal of Botany, 52: 209-222.

2014. Anuario forestal 2014. Boletín estadístico $N^{\circ} 144$. Instituto Forestal. Santiago, Chile, 159 p.
Klein, A.; Vaissiere, B.; Cane, J.; Steffan-Dewenter, I.; Cunningham, S.; Kremen, C.; Tscharntke, T.

2007. Importance of pollinators in changing landscapes for world crops. Proceedings of the Royal Society of London. Series B, 274: 303-313.

Lopes, L.; Blochtein, B.; Ott, A.

2007. Diversidade de insetos antófilos em áreas com reflorestamento de eucalipto, Município de Triunfo, Río Grande do Sul, Brasil. Iheringia, Série Zoología, 97(2): 181-193.

Majer, J.; Recher, H.

1999. Are eucalypts Brazil's friend or foe?. An entomological viewpoint. Anais da Sociedade Entomológica do Brasil, 28(2): 185-200.

Neves, F.; Braga, R.; Araújo, L.; Campos, R.; Fagundes, M. 2012. Differential effects of land use on ant and herbivore insect communities associated with Caryocar brasiliense (Caryocaraceae). Revista de Biología Tropical, 60(3): 1065-1073.

Pinto, R.; Junior, J.; Zanuncio, T.; Zanuncio, J.; Lacerda, M. 2004. Coleópteros coletados com Armadilhas luminosas em plantios de Eucalyptus urophylla na região amazônica brasileira. Ciência Floresta, 14(1): 111-119. 
Potts, B.M.

2004. Genetic improvement of Eucalyps. In: Encyclopedia of forest science. Burley, J.; Evans, J.; Youngquist, J.A. (eds.). Elsevier Science, Oxford, pp. 1480-1490.

Prys-Jones, O.; Corbet, S.A.

1991. Bumblebees. Richmond Publishing Co. England, 92 p. Rojas, P.

2014. Biología reproductiva e hibridación de Eucalyptus globulus. In: Mejoramiento genético de eucaliptos en Chile. Ipinza, R.; Barros, S.; Gutiérrez, B.; Borralho, N. (eds.). INFOR-FIA, Santiago, Chile, pp. 201-210.

\section{Sanhueza, R}

2014. Programa de mejoramiento genético de Eucalyptus nitens de SPT (Seed Production Technologies) Chile. In: Mejoramiento genético de eucaliptos en Chile. Ipinza, R.; Barros, S.; Gutiérrez, B.; Borralho, N. (eds.). INFOR-FIA, Santiago, Chile, pp. 477-488.

Santos, G.; Zanuncio, T.; Vinha, E.; Zanuncio, J.

2002. Influência de faixas de vegetação nativa em povoamentos de Eucalyptus cloeziana sobre população de Oxydia vesulia (Lepidoptera: Geometridae). Revista Árvore, 4(4): 499-504.

Sanzana, M.J.; Parra, L.; Benítez, H.; Espejo, J.

2012. Entomofauna polinizadora de Eucalyptus nitens en huertos semilleros del centro sur de Chile. Bosque, 33(1): $25-31$

Souto, X.; Bolaño, J.; González, L.; Reigosa, M.

2001. Allelopathic effects of tree species on some soil microbial populations and herbaceous plants. Biologia Plantarum, 44(2): 269-275.

Speight, M.; Hunter, M.; Watt, A.

1999. Ecology of insects concepts and applications. Oxford, Blackwell Science, New York, EE.UU. 350 p.

Tosso, J.

1985. Suelos volcánicos de Chile. Ministerio de Agricultura, Instituto de Investigaciones Agropecuarias. Santiago, Chile, $723 \mathrm{p}$.

Tscharntke, T.; Steffan-Dewenter, I.; Kreuss, A.; Thies, C. 2002. Characteristics of insect populations on habitat fragments: A mini review. Ecological Research, 17: 229-239. 